\section{H.C. Kogel \\ Die laparoskopische Anlage aortobifemoraler Bypässe. Eine neue Technik für ein bewährtes gefäßchirurgisches Rekonstruktionsverfahren}

Gefässchirurgie (1999) 4:34-39

\section{Kommentar zur Arbeit von L. Barbera, M. Kemen, V. Zumtobel, A. Mumme}

Der Fortschritt der minimal-invasiven Chirurgie stellt in der heutigen Zeit eine Herausforderung dar, neues Terrain zu erobern. Sicherlich werden Patienten langfristig enorm profitieren. In der vorliegenden Arbeit konnten an 16 Patienten gefäßchirurgisch Erfahrungen gesammelt werden mit sehr erfreulichen Ergebnissen. Es fallen jedoch einige technische Details auf, die diskussionswürdig sind.

Untersuchungen haben gezeigt, daß zentrale End-zu-Seit-Anastomosen bei konventionellen aortoiliakalen und -femoralen Rekonstruktionen im Langzeitverlauf komplikationsträchtiger als End-zu-End-Anastomosen sind. Die Bevorzugung des End-zu-Seit-Anschlusses an der infrarenalen Aorta bei der laparoskopischen Chirurgie resultiert aus rein technischen Erwägungen, da diese Anastomose leichter als die End-zuEnd-Anastomose herzustellen ist. Weiterhin zeigt Abb. 2 in Übereinstimmung mit dem Text, daß die Autoren offenbar keine lokale Exzision der Aortenwand vor Anastomosierung vornehmen. Hierdurch ergibt sich notwendigerweise ein Pseudoaneurysma, was im Langzeitverlauf zu erheblichen Problemen führen kann, wie mehrfach in großen Serien nachgewiesen werden konnte. Ein weiteres Problem ergibt sich technisch beim Durchziehen der Prothesenschenkel. Während bei schlanken
Patienten rechtsseitig ein Durchziehen der Prothesensschenkel mit der Kornzange hinter dem Ureter laparoskopisch sichtbar und damit möglich ist, entzieht sich auf der linken Seite die Kontrolle der Prothese in Bezug zum Ureter nach deren Durchziehen der Einsichtnahme wegen des Sigmas. Daher ist mit Kompressionskomplikationen des linken Ureters bei Fehlplazierung im Langzeitverlauf zu rechnen.

Ohne die operativen Leistungen der Autoren schmälern zu wollen, möchte ich darauf hinweisen, daß die bekannten technischen Fehler bei konventioneller Implantation von aortofemoralen Bifurkationsprothesen, die bei der offenen Technik diskutiert und mehrfach analysiert wurden, möglichst nicht in der minimalinvasiven Chirurgie wiederholt werden sollten. Hier würde das technisch Machbare langfristig die Qualität durch Spätkomplikationen schmälern.

H.C. Kogel

Dreifaltigkeits-Hospital, Klosterstraße 31, D-59555 Lippstadt

\section{Barbera \\ M. Kemen \\ V. Zumtobel \\ A. Mumme}

\section{Stellungnahme der Autoren}

Wir danken Herrn Prof. Kogel für die Anmerkungen zu einigen technischen Aspekten des vorgestellten laparoskopischen Verfahrens.

Einleitend möchten wir darauf hinweisen, daß die laparoskopische Operation nach denselben Prinzipien erfolgt, wie sie für die konventionelle Operation in unserem Hause gelten. Schon seit etwa 20 Jahren wählen wir routinemäßig einen proximalen End-zu-Seit-Anschluß für die Bifurkationsprothese. Die bei dieser
Vorgehensweise mögliche Erhaltung der A. mesenterica inf. sowie einer evtl. noch offenen A. iliaca interna sind unserer Meinung nach wichtige Vorteile des Bypassprinzips. Schwerwiegende intestinale Ischämien sind in unserem Krankengut auf diese Weise vermieden worden.

Eine lokale Exzision der Aortenwand, wie vom Autor gefordert, ist in unserem Krankengut ebenfalls nicht üblich, wäre aber auch bei der laparoskopischen Operation machbar. Ein Nahtaneurysma wurde in unserem Kollektiv nicht beobachtet. Lediglich beim Vorliegen einer dilatativen Atherosklerose würden wir den infrarenalen Anschluß in Endzu-End-Technik vornehmen.

Der retroperitoneale Durchzug der Prothesenschenkel gelingt laparoskopisch ähnlich sicher wie bei der konventionellen Operation. Beim schlanken Patienten kann der Durchzug des linken Prothesenschenkels durch Hochschlagen des Mesosigma laparoskopisch verfolgt werden. Bei adipösem Retroperitoneum besteht die Möglichkeit, die Tunnelierung unter videoskopischer Kontrolle von inguinal her vorzunehmen. Dabei gelingt es in aller Regel, die Kornzange lateral der Beckenstrombahn bis knapp oberhalb der Iliakabifurkation unter Sicht vorzuführen. Wenngleich nicht der gesamte Durchzug unter Sicht erfolgen kann, so ist das Risiko einer Fehlplazierung des Prothesenschenkels gering. In unserem Kollektiv von inzwischen fast 40 laparoskopisch operierten Patienten sind bislang derartige Komplikationen nicht aufgetreten.

Abschließend möchten wir uns bei Herrn Prof. Kogel für die Gelegenheit dieser zusätzlichen Diskussion bedanken.
L. Barbera $\bowtie \cdot$ M. Kemen · V. Zumtobel
A. Mumme
Chirurgische Klinik, Ruhr-Universität, Fachbereich Gefäßchirurgie,
St. Josef-Hospital, Gudrunstraße 56, D-44791 Bochum 